\title{
Attitudes et attentes des médecins généralistes à l'égard de la formation médicale continue
}

\author{
Ahmed BEN ABDE AZIZ, Sofiène HADDAD, Imed HARRABI, \\ Rafika GAHA, Hassen GHANNEM.
}

\begin{abstract}
Résuméc ontexte et but : La prise en cons dération des attentes des apprenants dans le domaine de la formation médicale continue est une condition préalableà sa réussite L'objectif de ce travail est d'étudier les perceptions et les atti tudes des médecins généralistes de la régi on sanitaire de Sousse (Tunisie) à l'égard de la nécessité, des buts, de l'obliga tion, de l'organisation et dela pédagogi e de la formation continue. M éthode :Il sagit d'une étude descriptive portant sur un collectif de 112 médecins généralistes exerçant dans les secteurs public et libéral, au cours de l'année 2000 (taux de participation : $80 \%$ ). Les données ont éé collectées à travers un questionnaire auto-administré Résultats :Selon cette étude, $24 \%$ des médecins généralistes ont été favorables à l'obligation légale de la formation médicale continue, $34 \%$ ont été favorables au principe de la recertification des titres scientifiques, mais $41 \%$ ont été hostiles à la participation d'un généraliste comme animateur. Conclusion :Les généralistes de la régi on de Sousse ont exprimé des attitudes asser compatibles avec une perception d'une formation médicale continue obligatoire, certifiée et active.
\end{abstract}

Mots clésformation médicale continue ; M édecine générale ; Attitudes; Tunisie.

Summary context and purpose :Takinginto cons deration the expectation of traineesin thedomain of continuing medical education is an essential condition to its success The objective of this work isto study the attitudes and the per ceptions of general practitioners in the region of Sousse (Tunisia) about the necessity, the goals, the obligation, and the organization of the current continuing medical education program. Method: It is a descriptive survey carried on 112 general practitioners working in the public and liberal sectors during the year 2000 (rate of participation rate: $80 \%$ ). Data were collected through a quetionnaire. Results:According to this survey, $24 \%$ of general practitioners were favo rable to the legal obligation of continuing medical education, $34 \%$ were favorable to the principle of the recertification of scientific titles, but $41 \%$ were against the involvement of a general practitioner as activity leader. Conclusion:General practitioners of the region of Sousse expressed attitudes which are in accordance with a perception of an mandatory, cer tified and active continuing medical education.

Keywordscontinuing medical education; General practice; Attitudes; Tunisia.

Pédagogie M édicale $2002 ; 3: 101-107$

\section{Introduction}

La formation médicale continue (FM C) est une étape dans le cursus d'une formation qui devrait se prolonger théoriquement à vie1. Son importance pour toutes les spécialités médicales fait actuellement consensus ${ }^{2}$ et son inscription dans la législation de plusieurs pays $^{3}$ au cours des dernières années, témoigne de sa nécessité non seulement pour développer les performances des praticiens ${ }^{4}$ mais aussi pour améliorer la qualité des soins dispensés aux patients et maîtriser les coûts ${ }^{5}$. La diversité des habiletés professionnelles des praticiens de première ligne et la transformation rapide des schémas diagnostiques et thérapeutiques expliquent la priorité qu'accordent les médecins généralistes à la FM C ${ }^{6}$.

Service d'Epidémiologie et de Statistiques Médicales - CHU Farhat Hached de Sousse (Tunisie).

Correspondance : Dr Ben Abdelaziz Ahmed - Service d'Epidémiologie et de Statistiques Médicales - CHU Farhat Hached de Sousse - Sousse 4000 - Tunisie.

Mailto:ahmed_epidemio@yahoo.fr 


\section{Recherche et Perspectives}

En Tunisie, la FM C, bien qu'elle soit non obligatoire sur le plan légal, connaît un développement rapide sur le plan quantitatif (environ 700 manifestations scientifiques sont annuellement organisées). Les initiatives sont multiples ${ }^{8}$ - provenant essentiellement du M inistère de la Santé Publique, des facultés de médecine, des associations médicales et du Syndicat des $M$ édecins de Libre Pratique - mais I'absence d'une coordination préalable et d'une évaluation a posteriori, rendent nécessaire une démarche qualité pour la FM C, garante de pertinence, de succès et d'efficience'.

La pertinence des activités de FM C pour les médecins généralistes est, aujourd'hui, un sujet de réflexion. $D$ 'une part, les experts nationaux de la pédagogie médicale ont douté de l'efficacité du modèle classique de l'enseignement post-universitaire centré sur l'enseignant : ils ont plaidé pour un modèle interactif centré sur l'apprenant ${ }^{10}$. $D$ 'autre part, les médecins généralistes ont exprimé leur insatisfaction devant la faible adéquation entre le contenu administré par les séances de FM C et leurs besoins spécifiques ${ }^{11}$.

L'étude des attentes des médecins général istes et de leurs résistances à l'égard de la FM C est une étape nécessaire pour la conciliation entre l'offre de formation et sa demande ${ }^{12}$. C e travail vise à identifier d'une part, les attitudes des médecins généralistes de la région de Sousse (Tunisie) à l'égard de la nécessité, des objectifs, de l'obligation, de l'organisation et de la pédagogie des séances de FM C et d'autre part, leurs facteurs de motivation pour la FM C.

\section{Matériel et méthode}

II sagit d'une étude descriptive et transversal e portant sur l'ensemble des médecins général istes du secteur public (exerçant dans les structures de soins relevant du M inistère de la Santé) et du secteur libéral (exerçant dans des cabinets privés) de la région sanitaire de Sousse (Tunisie) en I'an 2000. Cent quarante médecins éligibles ont été retenus en appliquant les critères d'inclusion suivants : être docteur en médecine, en activité libérale ou publique, en exercice dans les structures de première ligne de la région sanitaire de Sousse. O nt été exclus, les médecins stagiaires internes, les médecins en remplacement et les médecins généralistes attachés aux services spécialisés des hôpitaux universitaires de la région (ces médecins font fonction de spécialistes dans les hôpitaux et bénéficient de plusieurs opportunités de formation spécialisée, destinées aux médecins universitaires).

Les données ont été collectées à travers un questionnaire pré-testé et distribué par un médecin stagiaire interne aux praticiens généralistes au cours d'une visite directe à leurs cabinets ou à leurs centres de santé. Le questionnaire a été composé en majorité de questions de type fermé, couvrant plusieurs items relatifs aux attitudes des médecins généralistes à l'égard de la FM C (nécessité, objectifs, obligation, organisation, pédagogie) ainsi que les facteurs de motivation des médecins pour la FMC.

La sai sie et l'analyse des données ont été réal isées à I'aide du logiciel EPI IN FO (version 6).

Les statistiques descriptives (fréquence absolue, fréquence relative, moyenne) ont été utilisées pour les résumer. L'évaluation des attitudes des médecins a été effectuée selon une échelle de Likert à quatre propositions (Tout à fait d'accord, plutôt d'accord, pas vraiment d'accord, pas du tout d'accord). Au cours de l'analyse, les réponses des médecins ont été synthétisées en deux catégories. L'attitude a été jugée favorable pour les réponses : «tout à fait d'accord » et «plutôt d'accord » et non favorable pour les deux autres modalités.

\section{Résultats}

La population étudiée comporte 112 médecins généralistes de la région sanitaire de Sousse (année 2000). Q uarante sept (42\%) exerçaient dans le secteur libéral et $65(58 \%)$ appartenaient au secteur public. Vingthuit médecins (10 du secteur libéral et 18 du secteur publique) ont refusé de répondre au questionnaire, ramenant ainsi le taux de participation à $80 \%$. L'âge moyen des médecins interrogés a été de 41 ans avec une ancienneté professionnelle moyenne de 10 ans. Leurs attitudes à l'égard de la FM C ont été les suivantes:

\section{La nécessité de la FMC}

Soixante dix neuf pour cent des généralistes du secteur libéral et $83 \%$ du secteur public (soit $81 \%$ du total) pensaient qu'à la fin de la formation médicale initiale ( $F M I)$, le médecin général iste n'est pas parfaitement opérationnel dansl'exécution de son profil de poste et qu'il continue à rencontrer des difficultés dans sa pra- 
tique médicale. Cette FM I était peu orientée vers les problèmes de santé spécifiques à la première ligne, selon $70 \%$ des généralistes de libre pratique et $65 \%$ des généralistes du secteur publique (soit $67 \%$ des médecins de deux secteurs). Elle ne renforçait pas l'autonomie de l'apprenant dans la recherche de l'information scientifique sel on $62 \%$ des généralistes interrogés (soit $57 \%$ en privé et $66 \%$ en public). Un médecin généraliste du secteur public sur 5 et 1 médecin généraliste libéral sur 10 ont déclaré que le diplôme de "doctorat en médecine » a une validité limitée dans le temps ; $28 \%$ des praticiens étaient favorables à la recertification de leurs titres scientifiques.

\section{Les objectifs}

Le concept de la F M C a été jugé identique à la formation post-universitaire par $58 \%$ des médecins généralistes (soit $64 \%$ des médecins du secteur privé et $58 \%$ des généralistes du secteur publique). D 'après $54 \%$ des généralistes, ce concept était synonyme de formation professionnelle. Cette perception a été plus constatée dans le secteur public (58\%) que dans la pratique libérale (49\%).

Le tableau I montre que l'adaptation des praticiens aux spécificités de la pratique de la médecine générale et l'actualisation des connaissances médicales délivrées par la faculté étaient les deux objectifs essentiels de la F M C selon respectivement $93 \%$ et $92 \%$ des participants.

\section{L'obligation de la FMC}

Seulement $24 \%$ des médecins généralistes ont été favorables à l'obligation légale de la FM C (figure 1). C ette attitude était deux fois plus partagée par les médecins du secteur public que par leurs confrères de pratique libérale (31 contre $15 \%$ ).

\section{Les facteurs de motivation}

O utre, I'autosatisfaction personnelle, $72 \%$ des médecins du secteur public pensaient que la valorisation de la FM C dans les concours de promotion professionnelle serait le facteur motivant le plus important. L'attribution d'avantages fiscaux aux participants était un projet approuvé par $46 \%$ des praticiens du secteur public et $38 \%$ dans le secteur libéral.

\section{L'organisation}

Selon $79 \%$ des médecins généralistes, l'organisation de la FM C devrait être essentiellement du ressort de la faculté de médecine. La participation des associations et des syndicats des médecins généralistes a été proposée respectivement par $70 \%$ (surtout par les médecins du secteur publique : 72 contre $66 \%$ ) et $58 \%$ des répondants (particulièrement en privé : $66 \%$ contre $52 \%$ ). L'implication de l'industrie pharmaceutique dans la planification de la FM C a été déconseillée par $57 \%$ des interrogés.

\begin{tabular}{|c|c|c|c|}
\hline \multicolumn{4}{|c|}{$\begin{array}{c}\text { Tableau I : Les objectifs de la formation médicale continue (FM C) } \\
\text { selon } 112 \text { médecins généralistes exerçant dans la région sanitaire de Sousse } \\
\text { au cours de l'année } 2000 \text { (en pourcentage) }\end{array}$} \\
\hline O bjectifs de la FM C & $\begin{array}{l}\text { Secteur } \\
\text { libéral }\end{array}$ & $\begin{array}{l}\text { Secteur } \\
\text { public }\end{array}$ & Total \\
\hline - D évelopper la spécial ité de la M édecine G énérale & 87 & 96 & 93 \\
\hline - Promouvoir les compétences des médecins généralistes & 83 & 91 & 87 \\
\hline - Améliorer la qualité des soins fournis aux patients & 92 & 95 & 93 \\
\hline - C ontribuer à la résolution des problèmes de santé communautaire & 75 & 83 & 79 \\
\hline - Entretenir les connaissances médicales délivrées par la faculté & 83 & 78 & 80 \\
\hline - Actualiser les connaissances médicales initiales & 87 & 95 & 92 \\
\hline - A dapter les médecins général istes aux spécificités de la pratique médicale en 1 đ̛e ligne & 92 & 93 & 93 \\
\hline - Adapter les praticiens aux objectifs des programmes nationaux du ministère de la santé & 60 & 90 & 78 \\
\hline
\end{tabular}




\section{Recherche et Perspectives}

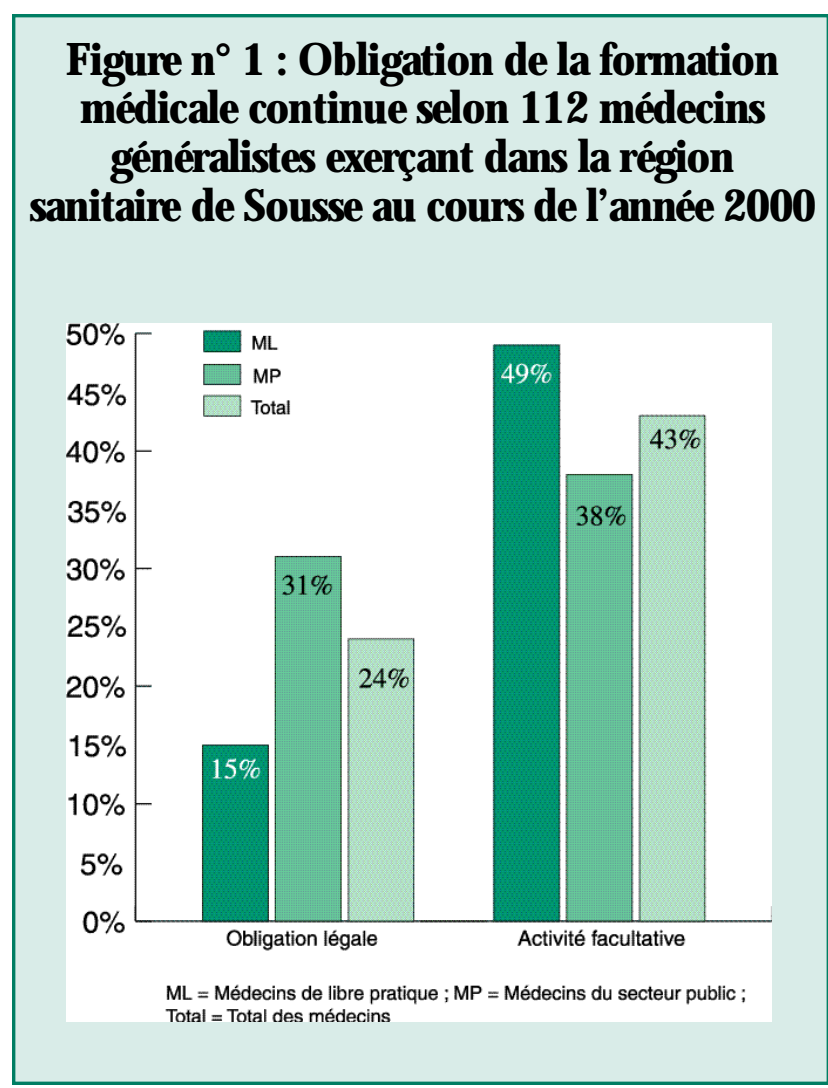

\section{La pédagogie}

La discussion en groupes (étude des cas cliniques) a été la principale méthode pédagogique proposée par les médecins généralistes ( $72 \%$ ) pour la FM C contrairement à la méthode magistrale et le stage hospitalier qui n'ont été indiqués que respectivement par $15 \%$ et $13 \%$ des omnipraticiens.

Q uatre vingt dix pour cent des médecins généralistes ont été favorables à l'organisation des séances de FM C sous la direction d'un médecin spécial iste universitaire expert du thème abordé. En revanche, la participation d'un médecin généraliste à l'animation des séances de FM C, à titre de formateur maîtrisant les aspects opérationnels des problèmes traités (en compagnie d'un expert spécialiste chargé de la clarification des éléments techniques trop complexes) et la réalisation des jeux de rôles n'ont été acceptées que respectivement par 49 et $34 \%$ des médecins généralistes.

\section{Discussion}

L'exploration des perceptions et des attentes des médecins général istes est l'étape préliminaire de la planification d'une FM C pertinente, adaptée à leurs besoins, rép ondant à leurs souhaits et par conséquent une FM $C$ efficace, réussie et durable 13. Ce travail a étudié les attitudes et les attentes des médecins généralistes à l'égard de la F M C.

M algré le taux de participation acceptable ( $80 \%$ ), ce travail n'a pas été à l'abri de quelques insuffisances méthodologiques (enquête régionale par questionnaire) limitant sa validité interne (le classique biais d'observation) et externe (difficulté de l'extrapolation à d'autres régions non universitaires).

II ressort de cette étude que les médecins généralistes

\section{Tableau II : Les facteurs de motivation de la formation médicale continue (FMC) d'après 112 médecins généralistes exerçant dans la région sanitaire de Sousse au cours de l'année 2000 (en pourcentage)}

\begin{tabular}{lccc}
\hline Facteurs de motivation à la FM C & $\begin{array}{c}\text { Secteur } \\
\text { libéral }\end{array}$ & $\begin{array}{c}\text { Secteur } \\
\text { public }\end{array}$ & Total \\
\hline $\begin{array}{l}\text { Etablir un lien entre la participation active à la FM C } \\
\text { et la revalidation du diplôme (recertification) }\end{array}$ & 13 & 26 & 20 \\
$\begin{array}{l}\text { Inciter financièrement les médecins généralistes participants } \\
\text { à la FM C en augmentant leurs honoraires ou leurs salaires }\end{array}$ & 13 & 45 & 31 \\
\hline $\begin{array}{l}\text { Accorder des avantages fiscaux aux participants réguliers } \\
\text { aux activités de la FM C }\end{array}$ & 38 & 46 & 43 \\
$\begin{array}{l}\text { Exiger la FM C dans tous les concours } \\
\text { de promotion professionnelle }\end{array}$ & 49 & 72 & 62 \\
\hline \begin{tabular}{l} 
L'autosatisfaction personnelle des médecins généralistes \\
\hline
\end{tabular} & 83 & 88 & 86
\end{tabular}

$M L=M$ édecins de libre pratique; $M P=M$ édecins du secteur public $; T=$ Total des médecins 
adhérai ent à trois principes de la FM C (la nécessité, la pédagogie active, la gouvernance universitai re) ; cependant, ils ont présenté trois réticences essentielles qui risquent d'engendrer une véritable résistance à une participation fructueuse aux activités de FM C (refus de l'obligation de la FM C, de la participation d'un général iste à son animation et de la recertification des titres universitaires).

\section{La FMC : nécessité sans obligation}

La nécessité de la FM C a étélargement partagée par les médecins général istes comme en témoigne leur perception d'une faible adéquation entre la formation médicale initiale (le curriculum) et le profil de poste (les compétences). C ette attitude s'explique d'une part, par l'incapacité du curriculum de l'enseignement médical à couvrir la complexité des situations pratiques de la souffrance humaine individuelle et communautaire et d'autre part, par la diversité des champs d'intervention de la médecine générale : restauration (curatif), protection (préventif) et promotion de la santé (éducatif). La motivation financière a été plus sollicitée par les généralistes du secteur public que par leurs confères du secteur libéral. En effet, les médecins salariés de la fonction publique, souhaitent accéder à travers la formation continue à une promotion professionnelle, leur rapportant plus de primes et d'avantages matériels. Les généralistes privés qui sont payés à l'acte et mieux honorés, semblent être moins sensibles à l'adoption d'une stratégie de motivation basée sur le revenu.

C ependant, chez les généralistes, la conception de la FMC est encore attachée au modèle classique de l'enseignement post universitaire ${ }^{14}$ dont l'objectif est d'améliorer les connaissances (pas les compétences) et le contenu est déterminé par l'enseignant expert (et non pas par l'apprenant).

En plus, le principe de l'obligation de la FM C a été refusé par les médecins généralistes de notre région contrairement à leurs confrères européens. U ne enquête de I'European Academy of Teachers in $G$ eneral $M$ edicine ${ }^{15}$ a montré que, sur les représentants des 20 pays étudiés, $55 \%$ ont proposé l'obligation de la FMC.

En Tunisie, la FM C est encore facultative, d'organisation disparate, sans contrôle et sans mesures légi sl atives incitatives ou obligatoires ${ }^{10}$. Son obligation est uniquement d'ordre déontologique et mora ${ }^{16}$. Alors qu'en
France, depuis le 24 avril 1996, une ordonnance gouvernementale a instauré pour la première fois l'obligation légale de la FM C ${ }^{2}$ (déà obligatoire dans 6 pays de la communauté européenne) ${ }^{17}$ sans pouvoir cependant l'appliquer concrètement jusqu'aujourd'hui.

\section{La FMC : une pédagogie active sans animation}

Les médecins généralistes ont plaidé pour l'adoption des méthodes pédagogiques actives: (discussion en groupe des cas cliniques) et ont souligné leur désir d'un échange et d'un accompagnement. En effet, la FM C devrait être considérée comme une opportunité d'échange des expériences entre les praticiens, de clarification de leurs difficultés et de recherche des conduites cliniques et thérapeutiques pertinentes. La pédagogie active n'est pas seulement efficace à la « rétention » des informations mais aussi adaptée aux adul tes ayant une expérience professionnelle large et diversifiée ${ }^{18}$.

Toutefois, une étude britannique ${ }^{12}$ concernant les médecins général istes a montré la supériorité des combinaisons entre présentation des cas et ateliers de travail en petits groupes. Selon une autre étude qualitative $^{19}$, les médecins ont perçu les rencontres conviviales au cours de la FM C , comme étant bénéfiques. Elles permettent une séparation entre la pratique médicale quotidienne et l'apprentissage qui devient d'autant plus efficace que l'ambiance est détendue et que les échanges d'idées entre confrères et superviseurs se font de façon informelle.

C ependant, les médecins généralistes de la région de Sousse ont préféré écouter passivement des experts chevronnés détaillant des questions spécialisées, refusant ainsi les méthodes interactives telles que les jeux de rôle et l'implication de leurs pairs à l'animation des rencontres : deux méthodes efficaces pour l'acquisition, l'intériorisation et l'expérimentation du savoir. Cette réticence à l'égard de la responsabilisation des généralistes dans I'animation des séances de formation destinées à leurs collègues pourrait être expliquée par l'ignorance de la faisabilité et de la pertinence de cette approche pédagogique d'une part, et leur crainte de la faible performance scientifique et didactique des animateurs non spécial istes et non universitaires d'autre part.

O $r$ au C anada, les séances de FM C des médecins de 


\section{Recherche et Perspectives}

famille se déroulent dans le cadre de petits groupes sous la direction d'un tuteur qui est aussi un médecin de famille ${ }^{20}$. En France ${ }^{21}$, la FM C, a été centrée sur la formation des animateurs appelés «leaders d'opinion ", chargés de relayer l'information pertinente auprès des utilisateurs potentiels. Un « leader d'opinion » dont l'impact positif sur la pratique médicale a été prouvé2z, est défini comme «un professionnel de santé désigné par ses collègues comme influent en terme de formation ».

\section{Une gouvernance universitaire sans recertification}

Les médecins interrogés attendent des facultés de médecine un rôle de leadership en matière de FM C. Certes, I'université pourrait offrir aux généralistes des ressources humaines et matérielles pour l'organisation des manifestations de $\mathrm{FM} \mathrm{C}$, mais surtout une crédibilité du contenu, trop attendue ${ }^{23}$.

L'engagement des facultés dans la FM C est une tradition très ancienne dans beaucoup des pays. $E n$ France, en plus de la contribution d'environ 1000 associations de généralistes dans la $F M C$, chaque faculté de médecine s'est dotée d'un département universitaire de formation continue des sciences de la vie et de la santéz ${ }^{23}$. Cependant la mauvaise connaissance dans l es facultés des réalités de l'exercice ambulatoire et l'absence de maîtrise de l'andragogie pourrait limiter leur impact dans la FMC des généralistes ${ }^{24}$.

C onjointement à cette attente, les médecins généralistes ont rejeté toute idée relative à la mise en ques-

\section{Références}

1.A yachi $\mathrm{K}$. La formation médicale continue: une chance pour la médecine au 3 e millénaire. Tunis $M$ ed $1999 ; 77(6 / 7): 308$.

2.D urieux P, Ravaud P, Chaix C, D urand-Zaleski I. La formation médicalecontinueamélioret-ellelecomporte ment des médecins? Presse M ed 1999 ; 28 (9):468-72.

3.Gallois P. La formation médicale continue, principes, organisation, objectifs, méthodes et évaluation. Paris: Flammarion-M édecine Sciences, 1997. tion de la validité de leurs titres universitaires telles que la recertification régulière des diplômes qui n'aurait été qu'un moyen de promotion de la qualité et non un instrument d'exclusion ou de protectionnisme corporatiste ${ }^{25}$. C e refus du principe de la recertification, présent égal ement chez les général istes européen $\mathrm{s}^{15}$ et les médecins de famille américain $\mathrm{s}^{26}$, illustre bien les résistances des médecins en exercice, à toute modification de la législation régulant la pratique médicale. Les prochaines générations seront sans doute plus réceptives au principe de la recertification des diplômes si elles sont prévenues de cette obligation dès l'enseignement prégradué.

\section{Conclusion}

II ressort de ce travail que les attitudes des général istes à l'égard de la FM C oscillent entre deux paradigmes: celui de l'enseignement post universitaire caractérisé par son centrage sur la transmission des connaissances par des experts avec un rôle passif des apprenants et celui de la formation professionnelle basée sur le transfert des compétences via une pédagogie active fai sant participer l'apprenant non seulement dans l'identification des besoins mais aussi dans l'animation des séances. La clarification du concept de la FM C , ses principes, ses objectifs et sa mission sont indispensable à toute réflexion sur les procédures de réalisation des séances de formation continue.

Le questi onnaire uti li sé dans cette étude peut être obtenu auprès du Dr Ben Abdelaziz

4.D avis D A. D oesC M E work ? An analysis of the effect of educational activities on physician performance or health care outcomes Int J Psychiatry M ed 1998 ; 28 : 21-39.

5.Ben Ammar M S, Ben Ameur M . La formation médi cale continue : étape d'une démarche dequalité !Tunis M ed $1999 ; 77(6 / 7): 327-37$

6.C antillon $\mathrm{P}$, Jones R. D oes continuing medical education in general practice makea difference? BM J 1999; $318: 1276-9$. 
7.Ben Salah $\mathrm{F}$, Ben Brahim H. Réflexion à propos de la formation médicale continue dans le secteur public. Tunis M ed $1999 ; 77$ (6/7) : 314-21.

8.Gueddena $B$, Ayachi K. L a Formation M édicale Continue dans le Secteur Privé. Tunis M ed $1999 ; 77$ (6/7) : 311-3.

9.Ayachi K. Etat actuel et problématique de la forma tion médicale continue en Tunisie. Tunis M ed 1999 ; 77 (6/7) : 370-1.

10.Essoussi AS, H achicha A, Chicha M, Ghannem H, Chaieb L. La FM C en Tunisie: Problématique et perspectives Conférence Internationale des $D$ oyens des Facultés de M édecine d'Expression Française.

http ://www.cidmef.ubordeaux2.fr/wnantes/texte9.htm

11. H addad Sofiène. Les attentes des médecins générali stes à l'égard de la formation médicale continue dans la régi on sanitaire de Sousse en 2000. Sousse: Faculté de M édecinel bn El Jazzar [thèse], 2001.

12.Kelly M H , M urray TS. General practionners' views on continuing medical education. $\mathrm{Br} J \mathrm{G}$ en Pract $1994 ; 44: 469-71$.

13.D avis $D A$, Thomson $M A, O$ xman $A D, H$ aynes $B$. Evidence for the effectiveness of CM E. JAM A $1992 ; 268$ : 1111-7.

14. Coles C. A review of learner-centered education and its applications in primary care E duc for Gen Pract $1991 ; 5,1: 19-25$.

15. WindaK A. A survey of current reaccreditation and recertification procedures in Europe. European Conference on Reaccredidation and Recertification. Cambridge; 24 et 24 mars 1995.

16. Brigley $\mathrm{S}$. Continuing medical education : the ques tion of evaluation. M ed Educ $1992 ; 26$ : 384-8.

17. Bignall $]$. Continung medical education shibboleth or necessity ? Lancet $1998 ; 351: 1004$.
18. $D$ avis $D A$, Thomson $M A, O$ xman $A D, H$ aynes RB. Changing physician performance : a systematic review of CME strategies. JAMA $1995 ; 274$ : 700-5.

19. Campion SC, Smith H, White P. Learners'experience of continuing medical education events: a qualita tive study of $\mathrm{GP}$ principals in D orset. Br J G en Pract $1998 ; 46: 1590-3$

20. Premi JN. Problem- Based, Self-D irected Continuing M edical Education in a Group of Practicing Family Physicians. J M ed Educ 1988 ; $63: 484-6$.

21. D rahi $E$, D uroux $G$. Formation à l'animation, la gestion des groupes, la pédagogie. In : Gallois $\mathrm{P}$, ed. La formation médicale continue, principes, organi sation, objectifs, méthodes et évaluation. Paris: Flammarion-M édecine Sciences, 1997.

22. Thomson M A, O xammn AD, H aynes RB. L ocal opinion leaders to improve health care professional practice and health care outcomes. The Cochrane Library 1998 .

(htpp :/www.cochrane.co.uk)

23. Conri C, Roland J, Boles JM. U niversité et forma tion médicale continue. In : GalloisP, éd. La forma tion médicale continue, principes, organisation, objectifs, méthodes et évaluation. Paris: Flammarion-M édecine Sciences, 1997.

24. Essoussi AS. Pratique professionnelle et Formation $M$ édicale Continue : La responsabilité des facultés de médecine. Tunis M ed $1999 ; 77$ (6/7) : 361-4.

25. Bensen JA. Certification and recertification: one approach to professional accountability. Ann Int M ed $1991 ; 114: 238-42$.

26. De Guise Vaillancourt M D. FM C : où en est-on outre-atlantique? Nouv Presse M ed 1981 ; 10:3027-33. 